\title{
An insight into the possible benefits of cupping therapy in COVID-19
}

\author{
Nooshin Abbasi $^{1}$, rezvan najafi², Azam Meyari² ${ }^{2}$, Mahdi Biglarkhani ${ }^{2}$ \\ 1 University of Padua \\ 2 Hamadan University of Medical Sciences
}

Funding: The author(s) received no specific funding for this work.

Potential competing interests: The author(s) declared that no potential competing interests exist.

\section{Abstract}

The coronavirus disease 2019 pandemic is caused by severe acute respiratory syndrome coronavirus 2 infection. The disease varies from asymptomatic or mild infection in the upper respiratory tract to severe respiratory syndromes and signs spread to the lower airways causing local inflammation and pneumonia. Currently no specific anti-virus drugs or certain vaccine without having any doubts on its short- or long-term adverse reactions in human body are approved to treat COVID-19. Patients affected by SARS in 2003 outbreak, with a $79.5 \%$ of shared sequence identity with SARS-CoV-2, benefited from traditional Chinese medicine. Many studies have demonstrated the benefits of herbal traditional chines medicines, acupuncture and moxibustion to manage COVID-19, but sparse studies have assessed the effects of cupping therapy in this pandemic. Cupping, specially its two main types wet and dry, seems to play a role in the activation of complement system, modulation of cellular parts of the immune system and in improvement of the majority of COVID-19 symptoms and signs. This study suggests that scientists and clinicians assess the therapeutic effects of cupping therapy as adjuvant treatment along with other therapeutic strategies in treatment of COVID-19. 


\section{The Effects of \\ Cupping Therapy CT on COVID-19}

COVID-19 abnormalities in lab. tests

CT may treat lymphopenia, high level of inflammatory markers \& adjust white blood cells count \& reduce ferritin, TNF- $a^{1}$, IL-6 ${ }^{2}$

COVID-19 common symptoms $\&$ signs

CT may treat fever, dry cough, headache, joint \& muscle pains, respiratory problems

\section{COVID-19 risk factors}

CT may prevent Diabetes, Obesity, Hypertension, Cardiovascular Diseases \& reduce blood glucose, ferritin, triglycerides, $\mathrm{LDL}^{3}$ cholesterol, $\mathrm{BMI}^{4}, \mathrm{SBP}^{5}$
1. TNF- a (tumor necrosis factor- $a$ )
2. IL-6 (interleukin-6)
3. LDL (low density lipoprotein)
4. BMI (body mass index)
5. SBP (systolic blood pressure)

The coronavirus disease 2019 (COVID-19) pandemic caused by severe acute respiratory syndrome coronavirus 2 (SARS-CoV-2) broke in December 2019 in Wuhan (Hubei, China). SARS-CoV-2 has been detected as the third coronavirus spread in humans that caused substantial morbidity and mortality 
through its intensive and rapid human to human transmission. The infection may be asymptomatic or it may cause a wide spectrum of symptoms and signs ${ }^{[1]}$. SARS-CoV-2 infection in severe cases may develop symptoms and signs in respiratory tract and sepsis in addition to cytokine storm and macrophage activation syndrome ${ }^{[2]}$. Unfortunately, specific antiviral drugs to cure or vaccine without having any doubts on its short- or long-term adverse reactions in the human body to prevent the infection have not been fully approved yet. Several studies have demonstrated the benefits of acupuncture ${ }^{[3][4][5]}$, moxibustion ${ }^{[6]}$ and herbal medicines such as Nigella sativa seed ${ }^{[7]}$ and at least 26 traditional Chinese medicine herbs with anti-novel coronavirus effects to manage the COVID-19 at different stages ${ }^{[8][9]}$, but sparse studies have assessed the effects of cupping therapy in this new pandemic ${ }^{[10]}$.

According to the National Center for Complementary and Integrative Health (NCClH), "Cupping therapy is a traditional practice, most notably in traditional medicine, which involves placing cups on the skin to create suction believed to help increase blood flow and promote healing associated with chronic neck and low back pain in the short term". Many studies have demonstrated promising preventive and therapeutic effects of cupping therapy in different pathological conditions rather than just pain including high fever due to infection of the upper respiratory tract, pulmonary dysfunctions in asthmatic children, type 2 diabetes mellitus, autoimmune diseases such as rheumatoid arthritis, hypertension, myocardial infarction and cardiac arrhythmias and chronic fatigue syndrome[11][12][13][14][15][16].

Cupping therapy may ameliorate the most common symptoms and signs of COVID-19.

Fever is among the most common signs observed at the beginning of COVID-19 in up to $90 \%$ of patients $^{[17]}$. Liu Ying Dong studied the antipyretic effect of dry cupping therapy on certain points based on meridian system according to Chinese traditional medicine in 103 cases to treat fever from $39^{\circ} \mathrm{C}$ to 39.99 ${ }^{\circ} \mathrm{C}$ in upper respiratory tract infection. After cupping therapy, the temperature dropped to $38.5^{\circ} \mathrm{C}$ after one hour, to $37^{\circ} \mathrm{C}$ after 6 hours, and to $36.8^{\circ} \mathrm{C}$ after 14 hours ${ }^{[11]}$.

Dry cough classifies as another most common early symptoms in COVID-19 in 60\%-86\% of patients ${ }^{[18]}$. Some studies in Chinese reported that moving cupping can relief asthma, cough, and muscle pain in the common cold ${ }^{[19][20][21]}$. Cupping therapy by stimulating meridian system may ameliorate cough in COVID19 patients.

Respiratory problems and shortness of breath is among the most common symptoms in $53 \%-80 \%$ of hospitalized COVID-19 patients ${ }^{[17]}$. Wet cupping therapy is recommended to relieve complications in respiratory disorders. A case study reported a recovery from acutely symptomatic COVID pneumonia with cupping therapy and acupuncture. The authors claims that specific lung meridian points such as LU 1 and LU 6, stimulate the respiratory system and ameliorate hypoxia and distress. These meridian points include the Mu point and the Xi-Cleft point that are associated with dyspnea and pleuritic chest pain in COVID$19^{[10]}$. Hong Jiaxuan et al. claimed that a combination of different types of dry cupping therapy in asthmatic children could improve the pulmonary functions including forced vital capacity (FVC), forced expiratory volume (FEV1), peak expiratory flow (PEF) and mid-expiratory flow (MEF) for 25\%-75\% ${ }^{[12]}$. A randomized controlled trial studied the effects of cupping therapy on arterial $\mathrm{O}_{2}$ saturation in smokers and 
found an increasing $\mathrm{O}_{2}$ saturation up to 12 hours with a better breathing expressed by patients after its application $^{[22]}$.

lymphopenia is the one of major problem in high percentage of patients affected by SARS-CoV-2 infection especially in severe cases. Natural killer (NK) cells destroy virally infected cells and together with cytotoxic T cells control the viral infection. Wet cupping therapy improves immunity through increasing white blood cells (WBCs) and NK cells ${ }^{[17]}$.

High levels of inflammatory markers such as ferritin, tumor necrosis factor- $\alpha$ (TNF- $\alpha$ ), IL-1 and IL- 6 is another abnormality in laboratory tests of patients. Cupping therapy suppresses immunopathogenesis through decreasing the serum level of autoantibodies, inflammatory mediators, and serum ferritin (a key player in autoimmunity). Studies showed that abnormal blood chemistry in autoimmune diseases can be restored by a pressure-dependent and size-dependent non-specific filtration of cupping therapy through arterial and venous end of capillaries ${ }^{[13]}$. A study evaluated the efficiency of wet cupping therapy as a complementary therapy in rheumatoid arthritis (RA) and found its immunomodulatory effects when is combined with the conventional medication therapy. Their results showed a significant increase in NK cells count and a markedly reduction of soluble interleukin-2 receptor (SIL-2R) concentration after 3 months of combined treatment compared to that of the sole conventional medication therapy ${ }^{[14]}$.

In conclusion, we hypothesize that cupping therapy as an adjuvant treatment along with other therapeutic strategies might ameliorate and cure COVID-19 symptoms and signs. However, cupping therapy is an almost easy and safe therapeutic method to practice but may not be applicable to all patients affected by COVID-19 specially in severe cases. The sequences, exact meridian points and the type, wet or dry, of the cupping may need to be studied precisely to treat the exact noted symptoms and signs and to prevent any side effects.

\section{References}

1. Na Zhu, Dingyu Zhang, Wenling Wang, Xingwang Li, et al. (2020). A Novel Coronavirus from Patients with Pneumonia in China, 2019. N Engl J Med, vol. 382 (8), 727-733. doi:10.1056/nejmoa2001017.

2. `W. Joost Wiersinga, Andrew Rhodes, Allen C. Cheng, Sharon J. Peacock, et al. (2020). Pathophysiology, Transmission, Diagnosis, and Treatment of Coronavirus Disease 2019 (COVID-19). JAMA, vol. 324 (8), 782. doi:10.1001/jama.2020.12839.

3. `Zhenzhen Han, Yang Zhang, Pengqian Wang, Qilin Tang, et al. (2021). Is acupuncture effective in the treatment of COVID-19 related symptoms? Based on bioinformatics/network topology strategy. doi:10.1093/bib/bbab110.

4. ^(2021). Efficacy and safety of acupuncture therapy for asymptomatic infection of COVID-19: A protocol for systematic review and meta-analysis: Retraction. doi:10.1097/md.0000000000025227.

5. `Qinwei Fu, Hui Xie, Li Zhou, Xinrong Li, et al. (2021). Traditional Chinese medicine auricular point acupressure for the relief of pain, fatigue, and gastrointestinal adverse reactions after the injection of novel coronavirus-19 vaccines: a structured summary of a study protocol for a multicentre, three-arm, 
single-blind, prospective randomized controlled trial. Trials, vol. 22 (1). doi:10.1186/s13063-021-051383.

6. `Yue Zhou, Xiao Yan, Fengjun Ma, Qingchang Xia, et al. (2021). Effects of moxibustion for COVID-19 convalescence. doi:10.1097/md.0000000000025389.

7. `Muhammad Fakhar-e-Alam Kulyar, Rongrong Li, Khalid Mehmood, Muhammad Waqas, et al. (2021). Potential influence of Nagella sativa (Black cumin) in reinforcing immune system: A hope to decelerate the COVID-19 pandemic. Phytomedicine, vol. 85 , 153277. doi:10.1016/j.phymed.2020.153277.

8. `Chang-quan Ling. (2020). Traditional Chinese medicine is a resource for drug discovery against 2019 novel coronavirus (SARS-CoV-2). Journal of Integrative Medicine, vol. 18 (2), 87-88. doi:10.1016/j.joim.2020.02.004.

9. `Hua Luo, Yan Gao, Jian Zou, Siyuan Zhang, et al. (2020). Reflections on treatment of COVID-19 with traditional Chinese medicine. Chin Med, vol. 15 (1). doi:10.1186/s13020-020-00375-1.

10. ${ }^{a}$, bStephanie I. Cheng. (2021). Medical Acupuncture as a Treatment for Novel COVID-19-Related Respiratory Distress: Personal Experience from a Frontline Anesthesiologist. Medical Acupuncture, vol. 33 (1), 83-85. doi:10.1089/acu.2020.1467.

11. ${ }^{a}$, biu Y et al.. (2002). Cupping therapy for 103 cases of high fever due to infection of the upper respiratory tract. J Trad Chin Med.

12. ${ }^{a}$, bHong J et al.. (2006). Effects of cupping therapy on the pulmonary functions in asthmatic children. J Trad Chin Med.

13. a, bHussam Baghdadi, Nada Abdel-Aziz, Nagwa Sayed Ahmed. (2015). Ameliorating Role Exerted by AlHijamah in Autoimmune Diseases : Effect on Serum Autoantibodies and Inflammatory Mediators. IJHS, vol. 9 (2), 203-227. doi:10.12816/0024129.

14. ${ }^{a}, \mathrm{~b}$ Ahmed SM et al.. (2005). Immunomodulatory effects of blood letting cupping therapy in patients with rheumatoid arthritis. Egypt J Immunol.

15. `Müzeyyen Arslan, Nesibe Yeşilçam, Duygu Aydin, Ramazan Yüksel, et al. (2014). Wet Cupping Therapy Restores Sympathovagal Imbalances in Cardiac Rhythm. The Journal of Alternative and Complementary Medicine, vol. 20 (4), 318-321. doi:10.1089/acm.2013.0291.

16. `xiu-dong Meng, Hao-ran Guo, Qing-ying Zhang, Xin Li, et al. (2020). The effectiveness of cupping therapy on chronic fatigue syndrome: A single-blind randomized controlled trial. Complementary Therapies in Clinical Practice, vol. 40 , 101210. doi:10.1016/j.ctcp.2020.101210.

17. a, b, cGuan WJ et al.. (2020). Clinical Characteristics of Coronavirus Disease 2019 in China. N Engl J Med.

18. `Su Eun Park. (2020). Epidemiology, virology, and clinical features of severe acute respiratory syndrome -coronavirus-2 (SARS-CoV-2; Coronavirus Disease-19). Clin Exp Pediatr, vol. 63 (4), 119-124. doi:10.3345/cep.2020.00493.

19. ^Han LX et al.. (1998). Observation of therapeutic effect of cupping therapy on muscle pain caused by wind-pathogen Tianjin. Journal of Traditional Chinese Medicine.

20. ^Huang GQ et al.. (2004). Clinical observation on therapeutic effect of moving cupping therapy on wind- 
cold type of common cold Chinese. Journal of Current Clinical Medicine.

21. `Zhou YM et al.. (1994). Moving cupping therapy on 100 cases of common cold. Zhongguo Zhen Jiu.

22. `Davood Hekmatpou, Latif Moeini, Salar Haji Nad Ali. (2013). The effectiveness of wet cupping vs. venesection on arterial $\mathrm{O} 2$ saturation level of cigarette smokers: A randomized controlled clinical trial. Pak J Med Sci, vol. 29 (6). doi:10.12669/pjms.296.3365. 